DOI:

\title{
BLENDED LEARNING IN THE LANGUAGE CLASSROOM
}

\author{
Valentina Reshetnikova \\ A Senior Teacher \\ Russian University of Transport \\ (Moscow, Russian Federation) \\ e-mail: valentina_ros@mail.ru
}

\begin{abstract}
Annotation. A mixed form of education is that educational Internet technologies are used to support traditional full-time education. In blended learning, various methods of delivering educational material are used, such as e-learning and more traditional instructorled learning. Learning organizations are currently combining online learning resources with classroom instruction or mixing the use of an independent workbook with individual instruction. Blended learning is important because it allows you to use different ways of learning and can satisfy different needs and learning styles. The method of working in tandem when learning a foreign language is considered. The effective use of a mixed form of teaching foreign languages allows you to increase the motivation of students to get good results in mastering a foreign language.
\end{abstract}

Keywords: remote, blended, learning, computer, Internet

\section{СМЕШАННОЕ ОБУЧЕНИЕ В ЯЗЫКОВОМ КЛАССЕ}

\author{
Валентина Решетникова \\ Старший преподаватель \\ Российский университет транспорта \\ (Москва, Российская Федерация) \\ e-mail: valentina ros@mail.ru
}

\begin{abstract}
Аннотация. Смешанная форма обучения заключается в том, что образовательные Интернет-технологии используются в качестве поддержки традиционного очного образования. В смешанном обучении используются различные методы доставки учебного материала, такие как электронное обучение и более традиционное обучение под руководством инструктора. Учебные организации в настоящее время объединяют онлайновые учебные ресурсы с обучением в классе или смешивают использование самостоятельной рабочей тетради с индивидуальным инструктажем. Смешанное обучение важно, так как оно позволяет использовать различные способы обучения и может удовлетворить различные потребности и стили обучения. Рассматривается метод работы в тандеме при изучении иностранного языка. Эффективное использование смешанной формы обучения иностранным языкам позволяет повысить мотивацию студентов получить высокие результаты в овладении иностранным языком.
\end{abstract}

Ключевые слова: дистанционный, смешанный, обучение, компьютер, Интернет

ВВЕДЕНИЕ. Современная методика выделяет ряд ключевых форм обучения. Это аудиторное обучение, предполагающее непосредственный контакт учащегося и обучающего; кейс-обучение, ориентированное на самостоятельную работу обучающихся, обеспеченное набором необходимых дидактических средств; обучение в сетях (e-learning), организуемое с помощью инструментальной среды, и т. д.

В эпоху, когда навыки работы с информационными и коммуникационными технологиями так важны, и так много совместной работы, совместного использования ресурсов, разработки контента и обучения осуществляется в цифровом, асинхронном и дистанционном режиме, маловероятно, что обучение студентов продолжится основываться исключительно на печатных учебниках и очных уроках, проводимых с шагом в 50 минут. Большинство педагогов, родителей и политиков считают «онлайн-обучение» подмножеством дистанционного обучения (где учащиеся и преподаватели географически разделены), в котором доставка контента и коммуникация осуществляются главным образом с использованием компьютеров, подключенных к Интернету.

В течение последних сорока лет индустрии обучения и развития постоянно внедряются новые инструменты, разные платформы и разные подходы к обучению. Благодаря лучшей и быстрой технологии мы начали использовать электронное обучение. Хотя электронное обучение было встречено с большими ожиданиями, теперь мы знаем больше о том, когда и как его использовать, и что наиболее эффективные программы электронного обучения - это те, которые сочетаются с другими учебными ресурсами.

В переводе с английского языка "blended learning" означает “смешанное обучение". Это система обучения, которая совмещает в себе элементы и подходы преподавания в аудитории, автономного и дистанционного обучения. Суть смешанной формы заключается в том, что образовательные Интернеттехнологии используются в качестве поддержки традиционного очного образования. Структура смешанного обучения включает в себя четыре основных составляющих: e-learning, т.е. обучение в Интернете, работа на компьютере с использованием интерактивных программ; самостоятельная работа - изучение грамматического материала, работа с текстами, словарем, развитие навыка восприятия на слух (аудио-, видеоресурсы); работа с 
преподавателем - индивидуальная и групповая работа под руководством преподавателя, практика общения и отработка изученного материала; коучинг - режим личных консультаций с преподавателем (телефон, е-таil).

ОБЗОР ЛИТЕРАТУРЫ. Появление обучения, которое сочетает в себе онлайн и личную доставку, является не просто теорией - оно уже разрабатывается и внедряется учебными заведениями по всему миру, а в некоторых случаях продолжается уже несколько лет. Смешанному обучению посвящены труды многих российских и зарубежных ученых. В России это Полат Е.С., Бухарина М.Ю., Моисеева М.В., Брейгина М.Е., Гетель Е.И., Бим И.Л.. Андреев, А.А., Солдаткин В.И., Тихомирова, Е.И., Шленов, Ю., Мосичева, И., Шестак. В. За рубежом вопросами дистанционного и смешанного обучения занимались Дзюбан, Хартман, Москаль, Дикельман, Стакер, Хорн, Вishop., Verleger.

МЕТОДЫ ИССЛЕДОВАНИЯ. Идея смешанного обучения состоит в том, чтобы синтезировать ряд различных подходов для создания эффективного обучения. В то время как смешанный подход не является новой концепцией, многие организации в настоящее время объединяют онлайновые учебные ресурсы с обучением в классе или смешивают использование самостоятельной рабочей тетради с индивидуальным инструктажем. Таким образом, организации максимизируют и оптимизируют использование ресурсов.

Этот комбинированный подход к использованию ресурсов и доказательство наличия связанных с обучением вариантов часто может улучшить усвоение учебного материала.

Онлайн-обучение может быть либо дистанционным, либо смешанным обучением, причем оба они поддерживаются новым надежным учебным подходом, в котором используются лучшие элементы обеих настроек. Независимо от точного определения смешанного обучения, все больше онлайн-школ и программ сочетают в себе онлайн-обучение и индивидуальное обучение.

Что такое смешанное обучение и как оно является частью дистанционного обучения? Существует много определений смешанного обучения (иногда называемого гибридным обучением), но в основном это сочетание онлайн-обучения и очного традиционного образования. Он берет лучшее из обоих миров и объединяет их в один курс. Смешанное обучение (Blended learning)— это форма обучения, при которой часть познавательной деятельности обучающихся проводится на знании под непосредственным руководством преподавателя, а часть деятельности выносится на самостоятельную индивидуальную работу обучающегося с электронными средствами обучения. Даже если образовательное учреждение не предоставляет никаких дистанционных курсов или чисто сетевых, обучающийся имеет возможность обучения в данной форме, если он посещает свое образовательное учреждение и получает материалы от другого учреждения или занимаясь самообразованием с использованием электронных средств обучения (Polat,.Bukharin, Moiseeva 2004:.5).

В научной литературе можно встретить несколько вариантов названия данной формы обучения: гибридное образование (Hybrid Learning), обучение посредством технологий (Technology-Mediated Instruction), Web-Enhanced Instruction (обучение посредством веб-ресурсов) и Mixed-Model Instruction (обучение в смешанном режиме). Смешанным признается еслии от 30 до 79\% учебного времени проводится онлайн.

Суть смешанного обучения заключается в том, что это не добавка к деятельности преподавателя, а замещение части его работы электронным ресурсом. Описываемые далее модели не относятся к смешанной форме обучения, однако в сочетании с онлайн обучением, могут представлять профессиональный интерес для преподавателей.

Традиционная модель очного обучения (Brick-and - mortar) предполагает работу в аудитории и делится на два вида: традиционное обучение (Traditional Instruction) и обучение с использованием электронных носителей информации (Теchnology- rich instruction). Traditional instruction- традиционная работа под непосредственным руководством преподавателя с использованием бумажных носителей информации по единому для всех расписанию. Тесhnology- rich instruction - традиционная работа под непосредственным руководством преподавателя с использованием электронных носителей информации (электронные доски, электронные книги, Интернет-инструменты и учебные планы в режиме сетевого доступа), без предоставления учебных материалов в сети. Следующие модели представляют собой два вида онлайн обучения, но в корне отличаются от смешанной формы обучения.

Informal online learning- неформальное, интегрированное дистанционное обучение на основе мультимедийных программ, когда студенты используют электронные средства обучения в свое свободное время, вне занятий. Например, образовательное видео игры или просмотр онлайновых лекций. Самоконтроль преобладает над со стороны преподавателя. Это могут быть также программы самообразования при возможности консультаций. Они также ориентированы на обучение взрослой аудитории, то есть тех, кто по каким-то причинам не смог закончить школьное образование.

Full-time online learning - сетевая модель обучения, когда студенты получают материалы через Интернет и взаимодействуют с преподавателем посредством Интернет- технологий на расстоянии. Тем не менее, такое обучение предполагает сдачу экзаменов, лабораторных работ непосредственно в очной форме обучения в присутствии преподавателя.

Концепция смешанного обучения представляет собой сочетание нескольких составляющих: дистанционного обучения (Distance Learning), обучение в аудитории (Face-to Face Learning), обучение через Интернет (Online learning), и самообучение (Self-learning). Иногда люди используют смешанное обучение означает объединение традиционного класса для одного курса и онлайн-курса для другого.

Мы все знаем, насколько эффективным может быть обучение в классе. Мы также знаем, насколько хорошо электронное обучение может обеспечить обучение базовым навыкам, процедурное обучение и моделирование для широкой и географически распределенной аудитории. Нельзя отрицать ценность обучения 
на рабочем месте, индивидуального обучения и наставника. Мы знаем сильные и слабые стороны этих ресурсов, поэтому мы пытаемся смешивать, используя наиболее эффективные компоненты каждого подхода. Таким образом, учащиеся получают лучшие биты от каждого ресурса, чтобы пройти соответствующую тренировку с высокой отдачей. Поэтому смешанное обучение представляет собой сочетание подходов к преподаванию и обучению. В смешанном обучении используются различные методы доставки, такие как электронное обучение и более традиционное обучение под руководством инструктора. Смешанное обучение важно, так как оно позволяет использовать различные способы обучения и может удовлетворить различные потребности и стили обучения.

Смешанное обучение - это не предоставление учащимся нескольких вариантов завершения обучения. Речь также не идет о предложении большого выбора или объединении подобных медиа для создания одного решения. Смешанное обучение - это смешение разных видов медиа и ресурсов для достижения оптимального решения для обучения. В конечном счете, точное определение смешанного обучения, помимо некоторой комбинации онлайн и очного обучения, может не иметь значения. В этой связи Дзюбан, Хартман и Москаль (2004) в кратком обзоре для EDUCAUSE под названием «Смешанное обучение» отметили, что смешанное обучение следует рассматривать как педагогический подход, сочетающий в себе эффективность и возможности социализации в классе с технологически улучшенными активными возможностями обучения.

В свое время, профессор Полат Е.С. утверждал, что «... дистанционное обучение - это новая форма обучения, которая существует в настоящее время уже во многих странах наряду с другими формами обучения очной, заочной экстернатом в системе непрерывного образования» (Polat,.Bukharin, Moiseeva 2004). Дистанционное обучение - взаимодействие преподавателя с обучающимися и обучающимися между собой, которое осуществляется на расстоянии и отражает все присущие учебному процессы компоненты: цели, содержание, методы, организационные формы, средства обучения, реализуемые специфичными средствами Интернет- технологий или другими средствами, предусматривающими интерактивность (Polat,.Bukharin, Moiseeva 2004).

Дистанционное обучение - это интерактивность, то есть постоянное взаимодействие преподавателя с обучающимися, и обучающимися между собой в учебном процессе, которое реализуется на двух уровня: обучающихся между собой и с используемыми ими средствами обучения. Ключевыми словами здесь являются «постоянное и систематическое взаимодействие на двух уровнях». Заочное обучение, это эпизодическое взаимодействие преподавателя с обучающимися и обучающихся с электронными средствами. Курс заочного обучения и курс дистанционного обучения принципиально отличаются один от другого организацией учебного материала, его структурой способом взаимодействия преподавателя и обучающихся, организацией информационно- образовательной среды учебного процесса, методами и формами обучения под воздействием используемых Интернет-технологий, встроенных в учебный процесс и представляющих его неотъемлемую часть. Система управления познавательной деятельностью обучающихся также обусловлена спецификой используемых услуг Интернет. Иными словами, взаимодействие возможно в рамках любой образовательной системы, но реализуется оно и познавательная деятельность обучающихся, специфичными средствами Интернет- технологий или других интерактивных технологий (Polat,.Bukharin, Moiseeva 2004).

В настоящее время большой акцент делается на самостоятельную познавательную деятельность студента, что немаловажно, так как во время обучения закладываются основы профессионализма и формируются умения самостоятельной профессиональной деятельности. Сокращение аудиторных занятий ведет к поиску новых путей оптимизации методов обучения и внедрения в учебный процесс новых технологий, которые бы способствовали формированию учебных умений студентов в их внеаудиторной самостоятельной работе. Самостоятельно приобретенные знания запоминаются лучше, особенно если студент лично заинтересован в получении знаний. Поэтому преподавателю нужно сделать все необходимое для того, чтобы представить студентам такую возможность. Одним из инструментов интенсификации учебно- методической работы преподавателя со студентами может стать смешанное обучение.

Смешивание может быть на уровне курса, сочетая как онлайн, так и не онлайн обучение в рамках одного предмета. Смешивание может быть на институциональном уровне, например, онлайн-школы собирают своих учеников на регулярной, запланированной основе, когда учитель физически присутствует или находится на расстоянии. Наконец, некоторые студенты посещают один или несколько полностью онлайн-курсов и посещают традиционные классы для одного или нескольких очных курсов, другого типа смешанной модели. Занятия в смешанном курсе можно представить в виде трёх циклов работы (обучения) - работа «до», работа «во время» и работа «после». Первый цикл обучения проходит в дистанционной форме. Студенты изучают теоретический материал, формируют базовые знания по тематике. В качестве подготовки используется определённое задание. Работа «во время» является синхронной - практические занятия и консультации - и требует подготовки и осмысления. Во время этого цикла преподаватель обсуждает задания студентов и их вопросы по теме, а также объясняет новую тему. По окончании занятия проводится закрепление и проверка полученных знаний при помощи тестов, вопросов или практических заданий. Последний цикл посвящен закреплению нового материала - выполнение домашнего задания, проекта и т.д. Учащиеся возвращаются к самостоятельному обучению: применяют все полученные знания на практике через интерактивные компоненты курса, выполняют задания, общаются между собой через средства виртуальных коммуникаций. Все три цикла повторяются неоднократно в течение одного курса или семестра обучения. Студенты учатся целенаправленно, не теряя времени на обсуждение и прослушивание ненужной или известной информации. Более того, если 
студенты в состоянии самостоятельно подготовиться к очередному занятию, то сами занятия становятся более интересными, так как появляется больше времени для практики, дискуссий и выполнения проектов

В последнее время не менее популярным становится изучение иностранных языков в Тандеме. Это форма открытого обучения, в котором люди с разными родными языками работают в парах. Цель Тандема овладение родным языком своего партнера в ситуации реального или виртуального общения, знакомство с его личностью, культурой страны изучае- мого языка, а также получение информации по интересующим областям знаний. Метод Тандема является одним из путей формирования речевой компетенции. Важнейшими принципами, раскрывающими суть метода, являются принципы обоюдности и автономии. Первый предполагает, что каждый из участников Тандема получит одинаковую пользу от общения. Второй основывается на том, что каждый из партнеров несет ответственность за конечный результат. В рамках данного обучения сочетаются традиционное (аудиторное) и виртуальное обучение на основе сетевых учебных курсов, то есть за «контактными» фазами следуют online-фазы. Такая форма объединяет преимущества обоих типов обучения. Это наличие прямых социальных контактов и, следовательно, развитие социальных компетенций при традиционном обучении, а также гибкость и эффективность электронного обучения, развитие информационных компетенций в ходе online - фаз.

Целью данной модели является совершенствование речевых навыков на коммуникативном и межкультурном уровне. В ходе учебного процесса происходит чередование не только виртуальных и реальных этапов работы в Тандеме, но и сам Тандем сменяется работой в одноязычных группах. На данном этапе уделяется особое внимание определенным грамматическим и лексикологическим вопросам и углубляется работа над языком. Кроме того, одноязычные этапы используются для организации двуязычных встреч дискуссий на актуальные темы или программ академической мобильности. Эффективность изучения иностранных языков в Тандеме зависит от совершенствования способностей к автономному обучению и того, как они используются в партнерстве.

Основными преимуществами Тандема признаются интерактивность процесса обучения; удобства, связанные с возможностью работать над учебным материалом в удобное время и в любом месте, где можно выйти в Интернет; возможность синхронной и асинхронной коммуникации с преподавателем и другими обучающимися; возможность реализации нелинейных, гибких концепций обучения; индивидуализация процесса обучения (индивидуальный темп, задания по выбору, учет индивидуальных интересов и склонностей); мультимедийность процесса обучения; активность всех каналов восприятия информации благодаря мультимедийности; возможность сочетать индивидуальные и групповые формы работы; открытость процесса обучения.

РЕЗУЛЬТАТЫ И ДИСКУССИЯ. Преимущества смешанного обучения, в первую очередь, заключаются в сочетании самостоятельного обучения с обучением в аудитории и дистанционным обучением. Это позволяет не только восполнить недостаток личного общения, но и учитывать все существующие стили обучения. То есть при смешанном обучении успехов могут достичь и те слушатели, которые лучше учатся самостоятельно, и те, которые лучше учатся со слов преподавателя.

Естественно, как и любая модель обучения, смешанная модель имеет свои недостатки. Наиболее характерный недостаток для данной модели - это необходимость детально прописать, что, как и когда будут делать учащиеся. Иначе есть риск получить в аудитории группу с совершенно разным уровнем знаний и низкий рейтинг завершения курса, так как большинство студентов сориентируется только на очное мероприятие, тем самым не получив большого объема знаний.

Смешанное обучение становиться все более популярным. Эффективность использования смешанной формы обучения иностранным языкам позволяет применять эту методику можно абсолютно к любой области знаний. Сегодня большинство западных компаний и учебных заведений активно используют эту модель в своей практике, регулярно проводя исследования, которые показывают только положительные аспекты сочетания традиционных и инновационных методов.,

ЗАКЛЮЧЕНИЕ. Таким образом, смешанное обучение можно рассматривать как систему, состоящую из единства ее компонентов педагогическое проектирование этой деятельности, ее содержательной и педагогической составляющих (в плане педагогических технологий, методов, форм обучения) и учебный процесс в сети. Дидактические особенности смешанной формы обучения, концепции образования в целом и обучения в частности определяют свою специфику формы подачи материала, отбора форм и средств обучения, для обеспечения эффективного решения задач подготовки специалистов.

\section{LIST OF REFERENCES}

E.S.Polat. M.Yu.Bukharin, M.V.Moiseeva; Pod. Red. E.S.Polat. - Teoriya i praktika distantsionnogo obucheniya: Uceb.Poobiye dlya stud. vissh. ped. Ucheb. Zavedeniy, M .: Izdatelskiy tsentr "Academiya", 2004, - 416 p.

For citation:

Reshetnikova Valentina (2019) BLENDED LEARNING IN THE LANGUAGE CLASSROOM // International ScientificPedagogical Organization of Philologists “WEST-EAST” (ISPOP). Scientific Journal WEST-EAST. Vol 2/1 N1 (October, 2019 ). p. 143-147. doi: 


\title{
Для цитирования:
}

Решетникова Валентина (2019) СМЕШАННОЕ ОБУЧЕНИЕ В ЯЗЫКОВОМ КЛАССЕ // Internationa 1Scientific-Pedagogical Organization of Philologists "WEST-EAST” (ISPOP) . Scientific Journal WEST-EAST. Vol 1/1 N1 (October, 2019). C. 143-147.. doi:

About the author: Reshetnikova Valrntina - a senior teacher of English and Russian as a forein language, the department "Foreign languages-4", the Institute of Transport Engineering and Control Systems, Russian University of Transport, Moscow, Russia.

e-mail: valentina ros@mail.ru

Сведения об авторе: Решетникова Валентина - старший преподаватель (английский язык и русский язык как иностранный), кафедра «Иностранные языки-4», Институт транспортной техники и систем управления, Российский университет транспорта, Москва, Россия.

e-mail: valentina ros@mail.ru

DOI:

\section{LANGUAGE TRAINING OF FOREIGN STUDENTS IN THE CONDITIONS OF BILINGUALISM}

\author{
Oksana Trostynska \\ PhD in Philology, Associate Professor \\ V.N. Karazin Kharkiv National University \\ (Kharkiv, Ukraine) \\ e-mail: okasana@ua.fm \\ Olena Kopylova \\ Senior Lecturer \\ V.N. Karazin Kharkiv National University \\ (Kharkiv, Ukraine) \\ e-mail: elene_k@i.ua
}

\begin{abstract}
The features of language training of foreign citizens at higher educational institutions of Ukraine are considered. The language situation in various regions of the country is analyzed. The models of teaching foreign students-non-philologists of learning the target language and the language of social and cultural communication in bilingualism conditions are described. A detailed analysis of the presented training models is given. The optimal model of language education for foreign students at Ukrainian universities is highlighted. The ways of solving the problems arising in the course of implementing the presented models of teaching inophones the Ukrainian and Russian languages are suggested. The usefulness of using a comparative method in teaching languages belonging to the same language group is denoted. The phenomena of positive and negative interference that arise in teaching the related languages are considered. The principles of teaching Russian language to inophones at V.N. Karazin Kharkiv National University are described. It is noted that the description of language material on the functional basis according to the principle "from the content to the form" allows structuring language material by combining the semantic content of speech with its formal organization. The competencies which are necessary for the formation of a Ukrainian-speaking language personality of a foreign student are considered.
\end{abstract}

Key words: bilingualism, foreign students, language learning models, higher educational institutions of Ukraine, students-nonphilologists 